\title{
Multichannel SAR Interferometry via Classical and Bayesian Estimation Techniques
}

\author{
Alessandra Budillon \\ Dipartimento per le Tecnologie, Università degli Studi di Napoli “Parthenope," via Acton 38, 80133 Napoli, Italy \\ Email: alessandra.budillon@uniparthenope.it
}

\section{Giancarlo Ferraiuolo}

Dipartimento di Ingegneria Elettronica e delle Telecomunicazioni, Università degli Studi di Napoli "Federico II," via Claudio 21, 80125 Napoli, Italy

Email: gferraiu@unina.it

Vito Pascazio

Dipartimento per le Tecnologie, Università di Napoli “Parthenope," via Acton 38, 80133 Napoli, Italy

Email:vito.pascazio@uniparthenope.it

\section{Gilda Schirinzi}

Dipartimento di Automazione Elettromagnetismo Ingegneria dell'Informazione Matematica Industriale (DAEIMI), Università degli Studi di Cassino, via Di Biasio 43, 03043 Cassino, Italy

Email:schirinzi@unicas.it

Received 10 August 2004; Revised 20 January 2005

\begin{abstract}
Some multichannel synthetic aperture radar interferometric configurations are analyzed. Both across-track and along-track interferometric systems, allowing to recover the height profile of the ground or the moving target radial velocities, respectively, are considered. The joint use of multichannel configurations, which can be either multifrequency or multi-baseline, and of classical or Bayesian statistical estimation techniques allows to obtain very accurate solutions and to overcome the limitations due to the presence of ambiguous solutions, intrinsic in the single-channel configurations. The improved performance of the multichannelbased methods with respect to the corresponding single-channel ones has been tested with numerical experiments on simulated data.
\end{abstract}

Keywords and phrases: synthetic aperture radar interferometry, statistical signal processing, Markov random fields.

\section{INTRODUCTION}

Synthetic aperture radar interferometric (InSAR) systems use more than one antenna (typically two), which can be displaced along the platform moving direction (along-track interferometry) or along the direction orthogonal to the moving direction (across-track interferometry). From the acquisitions of two or more image signals, these systems are able to recover additional information about the observed scene: they allow the reconstruction of the height profile of the earth surface in the across-track configuration $[1,2]$, and the detection of moving targets on the ground and the estimation of their range velocity in the along-track configuration $[3,4]$. This is possible because the interferometric phase, that is, the $(-\pi, \pi]$ wrapped phase of the signal obtained from the point-to-point correlation between the complex images acquired from the two interferometric antennas, is related the height values of the ground (for the across-track interferometry) and to the range velocity (for the along-track interferometry), through a known mapping. Then, after the so-called phase unwrapping (PhU) operation, a map of the ground elevation, for the first case, or of the target range velocity, for the latter case, can be retrieved.

As far as the across-track InSAR case is considered, various approaches [5] based on different strategies can be used to solve the height reconstruction problem. Among them, the ones based on least squares [6] and on network flow [7] are most used. They guarantee acceptable reconstruction results for regular height profiles, but they fail when height profiles exhibit high slopes and/or strong discontinuities, and in presence of low coherence values between the images acquired from the two SAR antennas, and in presence of phase jump larger than $\pi$ in the interferograms. In this last case, 
solution ambiguities are present. Moreover, they do not take into account the statistical properties of the noise present on the data (i.e., not Gaussian [2]). Other interferometric techniques using multipass acquisitions have been proposed in literature $[8,9,10]$.

In the along-track InSAR system, the problem is quite similar, but in this case only very few (typically only one) pixels of the image, the ones corresponding to the moving target, are involved. From one side, this simplifies the estimation procedure, from the other, it does not allow to introduce contextual information between adjacent pixel for increasing the estimation accuracy, like it happens in the across-track case. Moreover, other parameters besides coherence, such as the signal to clutter ratios, have to be considered. In the case of high signal-to-clutter ratio, the velocity can be easily recovered inverting a simple algebraic relation. When the signalto-clutter ratio decreases, the velocity estimation becomes gradually more and more critical, till it fails completely. Also in this case, as in the across-track case, there are solution ambiguities which can keep the velocity estimation from working correctly [11].

We propose to solve both above-mentioned problems by using statistical estimation methods, and exploiting multichannel interferograms. The statistical estimation methods allow to take into account from one hand the correct statistics of the involved noise (likelihood model); on the other hand, they can allow to model the same unknown of the problem as a random field (a priori statistical model). In such a way, it can be possible to design optimum estimation procedures. The use of a multichannel interferogram, which in this case can be obtained exploiting frequency diversity and/or baseline diversity, has a twofold effect: multichannel interferograms can help to reduce the variance of the estimation, and, if properly chosen, can allow to avoid solution ambiguities $[12,13,14]$.

In particular, for the across-track case, we describe a Bayesian estimation method formulated in terms of maximum a posteriori (MAP) estimation of the ground elevation profile. As a priori statistical description, capable of modeling pixels contextual information of the $2 \mathrm{D}$ height profile, a Gaussian Markov random field (GMRF) [15, 16] has been considered. This model is tuned and matched to the true height profiles thanks to the so-called hyperparameters [15] which can be estimated starting from the same measured wrapped phase data. In this situation, uniqueness is guaranteed by the use of multifrequency data. To take into account contextual information about the neighboring clusters and the use of multichannel interferograms allows the reconstruction of critical (for other PhU methods) profiles in the presence of nonnegligible noise.

As far as the along-track case is considered, a classical estimation method formulated in terms of maximumlikelihood (ML) estimation is adopted for the range-velocity estimation. Also in this case, the use of a multichannel configuration allows to estimate range velocities that cannot be estimated (because ambiguous) according to single-channelbased algorithms.



FIGURE 1: Across-track SAR interferometry geometry. An arbitrary point $P$ whose quota is $h$ is viewed from the two interferometric antennas with two different distances $R_{1}$ and $R_{2}$, respectively.

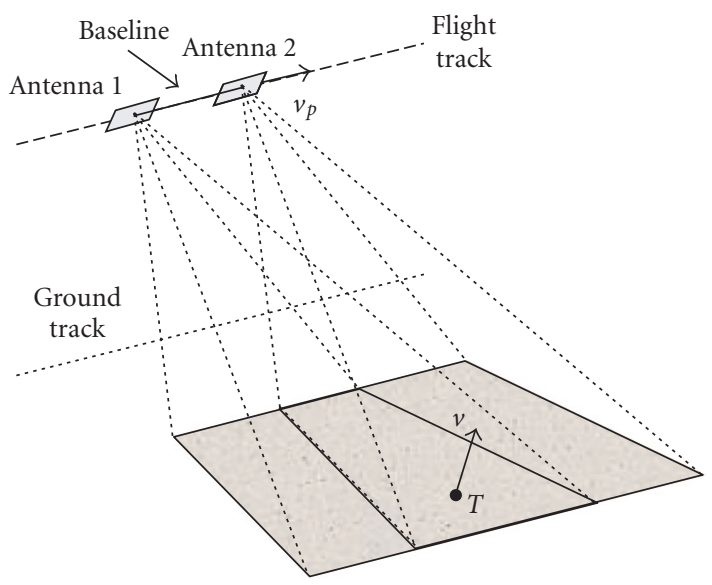

FIgURE 2: Along-track SAR interferometry geometry. A target $T$ moving on the ground, whose velocity is $\mathbf{v}$, is viewed with two different phase histories from the two interferometric antennas moving at velocity $\mathbf{v}_{p}$.

Some simulations and numerical experiments will also be presented to show and to validate the improved performance of multichannel-based methods with respect to singlechannel ones.

\section{SAR INTERFEROMETRIC SYSTEMS}

Consider in more detail the two different SAR geometries adopted for the across-track interferometry (Figure 1) and along-track interferometry (Figure 2).

Assuming a discrete ground coordinate system $(i, j), i=$ $1, \ldots, M, j=1, \ldots, P$, let $z_{1}(i, j)$ be the complex SAR image of a ground region acquired by the first antenna, and let 
$z_{2}(i, j)$ be the complex SAR image of the same ground region acquired by the second antenna.

The SAR interferometric signal is given by the complex correlation between the two SAR complex images:

$$
y(i, j)=z_{1}(i, j) z_{2}^{*}(i, j)
$$

and the SAR interferometric phase signal is given by

$$
\phi(i, j)=\tan ^{-1} \frac{\operatorname{Im}[y(i, j)]}{\operatorname{Re}[y(i, j)]}
$$

where $\operatorname{Re}[\cdot]$ and $\operatorname{Im}[\cdot]$ represent the real and imaginary parts of a complex quantity.

The phase values obtained by (2) using actual data are noisy. In the across-track case, their nominal (noise-free) values $\phi_{0}(i, j)$ contain the information about the quota values [2]:

$$
\phi_{0}(i, j)=\left\langle\frac{4 \pi d}{\lambda} h(i, j)\right\rangle_{2 \pi}
$$

where $\lambda$ is the wavelength corresponding to the working frequency $f=c / \lambda$ of the SAR system, $d$ is a parameter related to the orthogonal component of the baseline of the interferometric SAR system, and $\langle\cdot\rangle_{2 \pi}$ represents the "modulo- $2 \pi$ " operation.

In the along-track case, the interferometric phase nominal values $\phi_{v}(i, j)$ contain the information about $v_{r}$, the range component of the object velocity (orthogonal and coplanar to the platform motion direction) [3]:

$$
\phi_{v}(i, j)=\left\langle\frac{4 \pi b}{\lambda} \frac{v_{r}(i, j)}{\left|\mathbf{v}_{p}\right|}\right\rangle_{2 \pi}=\left\langle\frac{4 \pi b}{\lambda} u_{r}(i, j)\right\rangle_{2 \pi},
$$

where $b$ is a quantity related to the parallel component of the baseline, $\mathbf{v}_{p}$ is the velocity of the flying platform, and the normalized range velocity $u_{r}$ has been introduced, where the moving target is present, $v_{r} \neq 0$ and consequently $\phi_{v}(i, j) \neq$ 0 , otherwise the along-track inteferometric phase (4) is null.

The actual interferometric phase signal $\phi(i, j)$ (also called interferogram) differs from the nominal values given by (3) and (4) for the presence of phase noise. Also for the phase noise there are differences between the across-track and along-track cases which will be discussed in the following sections.

\subsection{Across-track interferometric phase first-order probability density function}

In the across-track case, the first-order probability density function (pdf) of the interferometric phase $\Phi$ is given by the well-know expression [17] (in the following we will use capital letters to denote random variables):

$$
\begin{aligned}
& f_{\Phi}\left(\phi ; \phi_{0}, \gamma\right) \\
&= \frac{1}{2 \pi} \frac{1-|\gamma|^{2}}{1-|\gamma|^{2} \cos ^{2}\left(\phi-\phi_{0}\right)} \\
& \times\left\{1+\frac{|\gamma| \cos \left(\phi-\phi_{0}\right) \cos ^{-1}\left[-|\gamma| \cos \left(\phi-\phi_{0}\right)\right]}{\left[1-|\gamma|^{2} \cos ^{2}\left(\phi-\phi_{0}\right)\right]^{1 / 2}}\right\}, \\
& \phi \in(-\pi, \pi],
\end{aligned}
$$

where $\gamma$ is the coherence coefficient representing the correlation between images $z_{1}(\cdot)$ and $z_{2}(\cdot)$ at $(i, j)$ and $\phi_{0}$ is the nominal (noise-free) phase defined in (3). The local dependence of $\phi, \phi_{0}$, and $\gamma$ on $(i, j)$ has been omitted to preserve the notation simplicity.

Some plots of pdf (5) for $\phi_{0}=0$ and for five different values of $\gamma$ are shown in Figure 3. The larger the coherence value (its maximum is one) the more peaked the pdf. For different values of $\phi_{0} \neq 0$, the curve maximum is located on $\phi_{0}$.

To restore a better physical meaning, pdf (5) can be rewritten substituting (3) in (5), and exploiting the $2 \pi$ periodic nature of the cosine function:

$$
f_{\Phi}(\phi \mid h ; \lambda, d, \gamma)=\frac{1}{2 \pi} \frac{1-|\gamma|^{2}}{1-|\gamma|^{2} \cos ^{2}(\phi-(4 \pi d / \lambda) h)}\left\{1+\frac{|\gamma| \cos (\phi-(4 \pi d / \lambda) h) \cos ^{-1}[-|\gamma| \cos (\phi-(4 \pi d / \lambda) h)]^{1 / 2}}{\left[1-|\gamma|^{2} \cos ^{2}(\phi-(4 \pi d / \lambda) h)\right]}\right\},
$$

Equation (6) has been written as a conditional pdf, pointing out that the quota $h$ can be viewed in its turn as a random parameter.

Note that also for $h=0$ (the case of a pure phase noise) the probability density corresponding to nonzero phase values is different from zero. This happens when the coherence $\gamma$ between the two SAR images acquired from the two antennas is less than one. A coherence loss in InSAR images can be due to the variations of the ground clutter when observed with two different angles and to the thermal noise at the receivers. Finally, note that in (6) the dependence on the wavelength $\lambda$ and on the baseline $d$ has been made explicit. 




FIGURE 3: pdf of the interferometric phase for $\phi_{0}=0$ and $\gamma=$ $0.1,0.3,0.5,0.7$, and 0.9 .

It will be a useful result in the following sections, when the multichannel algorithm will be discussed.

\subsection{Along-track interferometric phase first-order probability density function}

Let $Z_{c 1}=Z_{c 1 r}+j Z_{c 1 i}$ and $Z_{c 2}=Z_{c 2 r}+j Z_{c 2 i}$ be the clutter processes representing the SAR complex images acquired from the two antennas in the along-track SAR interferometry case.

It is well known that the SAR image of most natural scenes is a (complex) random process, whose real and imaginary parts are Gaussian, with zero mean and same variance, and uncorrelated [18], since they are resulting from the superposition of the signals backscattered from many scattering centers lying in the resolution cell. Then the joint probability density function of $\mathbf{Z}_{c}=\left[\begin{array}{llll}Z_{c 1 r} & Z_{c 1 i} & Z_{c 2 r} & Z_{c 2 i}\end{array}\right]^{T}$ is Gaussian, that is,

$$
\begin{aligned}
f_{Z_{c}}\left(z_{c}\right) & =f_{Z_{c 1 r}, Z_{c 1 i}, Z_{c 2 r}, Z_{c 2 i}}\left(z_{c 1 r}, z_{c 1 i}, z_{c 2 r}, z_{c 2 i}\right) \\
& =\frac{1}{(2 \pi)^{2}\left|\mathbf{C}_{c}\right|^{1 / 2}} \exp \left\{-\frac{1}{2} z_{c}^{T} \mathbf{C}_{c}^{-1} \mathbf{z}_{c}\right\}
\end{aligned}
$$

where $\mathbf{C}_{c}$ is the covariance matrix of real processes $Z_{c 1 r}, Z_{c 1 i}$, $Z_{c 2 r}$, and $Z_{c 2 i},\left|\mathbf{C}_{c}\right|$ is the determinant of $\mathbf{C}_{c}$, and $\mathbf{C}_{c}^{-1}$ is the inverse of $\mathbf{C}_{c}$. The column vector $\mathbf{z}_{c}$ is defined as

$$
z_{c}=\left[\begin{array}{llll}
z_{c 1 r} & z_{c 1 i} & z_{c 2 r} & z_{c 2 i}
\end{array}\right]
$$

Since $\mathbf{Z}_{c}$ is a zero-mean random vector, the covariance matrix $\mathbf{C}_{c}$ is defined as

$$
\begin{aligned}
\mathbf{C}_{c} & =E\left[\mathbf{Z}_{c} \mathbf{Z}_{c}^{T}\right] \\
& =\left[\begin{array}{llll}
\operatorname{Var}\left(Z_{c 1 r}\right) & E\left[Z_{c 1 r} Z_{c 1 i}\right] & E\left[Z_{c 1 r} Z_{c 2 r}\right] & E\left[Z_{c 1 r} Z_{c 2 i}\right] \\
E\left[Z_{c 1 i} Z_{c 1 r}\right] & \operatorname{Var}\left(Z_{c 1 i}\right) & E\left[Z_{c 1 i} Z_{c 2 r}\right] & E\left[Z_{c 1 i} Z_{c 2 i}\right] \\
E\left[Z_{c 2 r} Z_{c 1 r}\right] & E\left[Z_{c 2 r} Z_{c 1 i}\right] & \operatorname{Var}\left(Z_{c 2 r}\right) & E\left[Z_{c 2 r} Z_{c 2 i}\right] \\
E\left[Z_{c 2 i} Z_{c 1 r}\right] & E\left[Z_{c 2 i} Z_{c 1 i}\right] & E\left[Z_{c 2 i} Z_{c 2 r}\right] & \operatorname{Var}\left(Z_{c 2 r}\right)
\end{array}\right] .
\end{aligned}
$$

Since $Z_{c 1}$ and $Z_{c 2}$ are the lowpass representations of narrowband signals it can be assumed that $E\left[Z_{c 1 r} Z_{c 1 i}\right]=$ $E\left[Z_{c 2 r} Z_{c 2 i}\right]=0, E\left[Z_{c 1 r} Z_{c 2 r}\right]=E\left[Z_{c 1 i} Z_{c 2 i}\right], E\left[Z_{c 1 i} Z_{c 2 r}\right]=$ $-E\left[Z_{c 1 r} Z_{c 2 i}\right](E[\cdot]$ is the statistical average operator), and $\operatorname{Var}\left(Z_{c 1 r}\right)=\operatorname{Var}\left(Z_{c 1 i}\right)=\operatorname{Var}\left(Z_{c 2 r}\right)=\operatorname{Var}\left(Z_{c 1 i}\right)=\sigma^{2}$. Considering the coherence of the clutter $\gamma_{c}$ (i.e., the complex correlation coefficient of $Z_{c 1}$ and $Z_{c 2}$ ), defined as

$$
\begin{aligned}
\gamma_{c} & =\frac{E\left[Z_{c 1} Z_{c 2}^{*}\right]}{\left[\operatorname{Var}\left(\left|Z_{c 1}\right|\right) \operatorname{Var}\left(\left|Z_{c 2}\right|\right)\right]^{1 / 2}} \\
& =\frac{E\left[Z_{c 1 r} Z_{c 2 r}\right]+j E\left[Z_{c 1 i} Z_{c 2 r}\right]}{\sigma^{2}}=\frac{q}{\sigma^{2}},
\end{aligned}
$$

we derive

$$
\mathbf{C}_{c}=\left[\begin{array}{cccc}
\sigma^{2} & 0 & \operatorname{Re}(q) & -\operatorname{Im}(q) \\
0 & \sigma^{2} & \operatorname{Im}(q) & \operatorname{Re}(q) \\
\operatorname{Re}(q) & \operatorname{Im}(q) & \sigma^{2} & 0 \\
-\operatorname{Im}(q) & \operatorname{Re}(q) & 0 & \sigma^{2}
\end{array}\right]
$$

The presence of thermal noise at the receivers can be formalized considering two additive (to the clutter) zeromean Gaussian complex processes $N_{1}=N_{1 r}+j N_{1 i}$ and $N_{2}=N_{2 r}+j N_{2 i}$, independent of each other, and independent of the clutter processes, with $\operatorname{Var}\left(N_{1 r}\right)=\operatorname{Var}\left(N_{1 i}\right)=$ $\operatorname{Var}\left(N_{2 r}\right)=\operatorname{Var}\left(N_{2 i}\right)=\sigma_{n}^{2}$ and covariance matrix $\mathbf{C}_{n}=\sigma_{n}^{2} \mathbf{I}$. The two new processes representing the SAR images in presence of thermal noise $Z_{c 1}+N_{1}$ and $Z_{c 2}+N_{2}$ will have a covariance matrix equal to

$$
\mathbf{C}_{c}+\mathbf{C}_{n}=\left[\begin{array}{cccc}
\sigma^{2}+\sigma_{n}^{2} & 0 & \operatorname{Re}(q) & -\operatorname{Im}(q) \\
0 & \sigma^{2}+\sigma_{n}^{2} & \operatorname{Im}(q) & \operatorname{Re}(q) \\
\operatorname{Re}(q) & \operatorname{Im}(q) & \sigma^{2}+\sigma_{n}^{2} & 0 \\
-\operatorname{Im}(q) & \operatorname{Re}(q) & 0 & \sigma^{2}+\sigma_{n}^{2}
\end{array}\right]
$$

The main effect of thermal noise amounts to reduce the coherence, now given by

$$
\begin{aligned}
\gamma & =\frac{E\left[\left(Z_{c 1}+N_{1}\right)\left(Z_{c 2}+N_{2}\right)^{*}\right]}{\left[\operatorname{Var}\left(\left|Z_{c 1}+N_{1}\right|\right) \operatorname{Var}\left(\left|Z_{c 2}+N_{2}\right|\right)\right]^{1 / 2}} \\
& =\frac{E\left[Z_{c 1 r} Z_{c 2 r}\right]+j E\left[Z_{c 1 i} Z_{c 2 r}\right]}{\sigma^{2}+\sigma_{n}^{2}} \\
& =\frac{q}{\sigma^{2}\left(1+\sigma_{n}^{2} / \sigma^{2}\right)}=\frac{\gamma_{c}}{\left(1+\sigma_{n}^{2} / \sigma^{2}\right)} .
\end{aligned}
$$

Being the clutter and the noise processes stationary and zero mean, the term $\sigma_{n}^{2} / \sigma^{2}$ represents the ratio between the power of the thermal noise and the power of the clutter. Its inverse $\sigma^{2} / \sigma_{n}^{2}$ can be defined as the clutter-to-noise ratio (CNR).

Note that the above-derived model relative to the presence of clutter and additive thermal noise can be also applied to the derivation of the first-order pdf relative to the acrosstrack case given in (5). 




FIGURE 4: pdf of the interferometric phase in absence of moving target $\left(\phi_{v}=0\right)$, for three values of $\operatorname{CNR}(0,10,20 \mathrm{~dB})$, and $\gamma_{c}=0.95$.

In presence of a moving target, the SAR complex images become

$$
\begin{aligned}
& Z_{1}= \begin{cases}Z_{c 1}+N_{1}+z_{T 1} & \text { in presence of moving target, } \\
Z_{c 1}+N_{1} & \text { in absence of moving target }\end{cases} \\
& Z_{2}= \begin{cases}Z_{c 2}+N_{2}+z_{T 2} & \text { in presence of moving target, } \\
Z_{c 2}+N_{2} & \text { in absence of moving target, }\end{cases}
\end{aligned}
$$

where $z_{T 1}$ and $z_{T 2}$ denote the SAR (deterministic) images of the moving target relative to the two interferometric antennas (approximately 2D sinc signals in case of concentrated target).

The two processes $Z_{1}=Z_{1 r}+j Z_{1 i}$ and $Z_{2}=Z_{2 r}+j Z_{2 i}$ are still Gaussian, have mean (due to the moving target) different from zero:

$$
\begin{aligned}
\mathbf{m}_{Z} & =E\left[\begin{array}{llll}
Z_{1 r} & Z_{1 i} & Z_{2 r} & Z_{2 i}
\end{array}\right]^{T} \\
& =\left[\begin{array}{llll}
\operatorname{Re}\left(z_{T 1}\right) & \operatorname{Im}\left(z_{T 1}\right) & \operatorname{Re}\left(z_{T 2}\right) & \operatorname{Im}\left(z_{T 2}\right)
\end{array}\right]^{T},
\end{aligned}
$$

and same covariance matrix of the clutter-plus-noise process given by $\mathbf{C}_{z}=\mathbf{C}_{c}+\mathbf{C}_{n}$. The interferometric signal is finally given by the product $Z_{1} Z_{2}^{*}=A \exp (j \Phi)$.

The adopted model provides in absence of moving targets the pdf of the interferometric phase shown in Figure 4. Note that this pdf can also be obtained analytically from (5) setting $\gamma=\gamma_{c} /(1+1 / \mathrm{CNR})$, as shown in (13).

In presence of moving targets, the interferometric phase pdf depends on the CNR, on the coherence $\gamma_{c}$, on the signalto-clutter ratio (SCR), defined as the ratio between the intensity of the moving target and the power of the background clutter in the resolution cell, and also on the target range

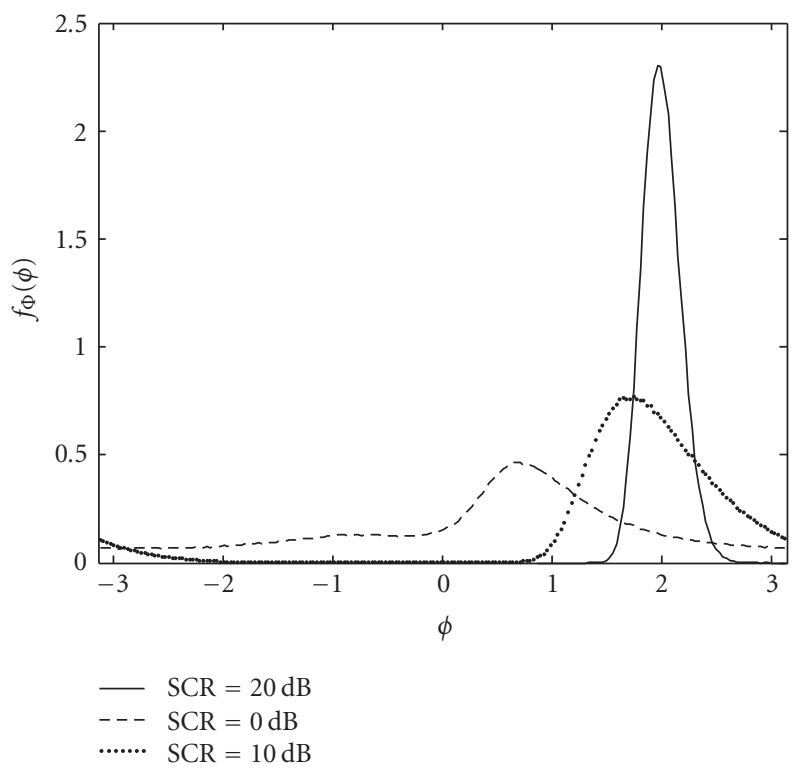

FIGURE 5: pdf of the interferometric phase in presence of a moving target such that $\phi_{v}=2.0 \mathrm{rad}$, for three values of $\operatorname{SCR}(0,10,20 \mathrm{~dB})$, $\mathrm{CNR}=10 \mathrm{~dB}$, and $\gamma_{c}=0.95$.

velocity through (4). Note that in along-track InSAR applications, the coherence is close to one. The statistical description of the interferometric phase $\Phi$ can be derived numerically via Monte Carlo techniques.

The pdfs of the interferometric phase evaluated for a range velocity such that the nominal value is $\phi_{v}=2.0 \mathrm{rad}$, for three values of $\operatorname{SCR}(0,10,20 \mathrm{~dB})$, for $\mathrm{CNR}=10 \mathrm{~dB}$, and a clutter coherence $\gamma_{c}=0.95$, are shown in Figure 5 .

Despite the lack of availability of an analytical form of this interferometric phase pdf, it is possible to make reasonings under the hypothesis of high and low SCRs. In the first case, the signal components $z_{T 1}$ and $z_{T 2}$ prevail over to clutter-plus-noise components. Consequently, $Z_{1} \approx z_{T 1}$ and $Z_{2} \approx z_{T 2}$ and $Z_{1} Z_{2}^{*}=z_{T 1} z_{T 2}^{*}=A_{T}^{2} \exp \left(j \phi_{v}\right)$, where $A_{T}^{2}$ is the moving target power. The overall random phase $\Phi$ of the interferometric signal tends toward the deterministic constant $\phi_{v}$, and its pdf tends toward a Dirac delta (see Figure 6). On the other hand, in the case of low SCR values, the clutter-plus-noise components prevail against the signal components, the interferometric signal tends toward the one relative to the absence of a moving target, and the phase pdf can be obtained as in Figure 4 setting the proper value of $\gamma_{c}$ and CNR. A numerical confirmation of the above discussion is shown in Figure 6, where the pdfs relative to SCR $=60 \mathrm{~dB}$ (high) and SCR $=-20 \mathrm{~dB}$ (low) are reported.

It has to be noted that the probability density function of the interferometric phase in presence of moving targets (see Figure 5) is not given by the shift, of a quantity equal to $\phi_{v}$, of the pdf in absence of moving target (see Figure 4), as sometimes reported in literature. For the pdfs reported in Figure 5, only the one relative to $\mathrm{SCR}=20 \mathrm{~dB}(\mathrm{CNR}=$ $\left.10 \mathrm{~dB}, \gamma_{c}=0.95\right)$ is centered on $\phi_{v}$, but its values are very different from the ones relative to the curve corresponding 


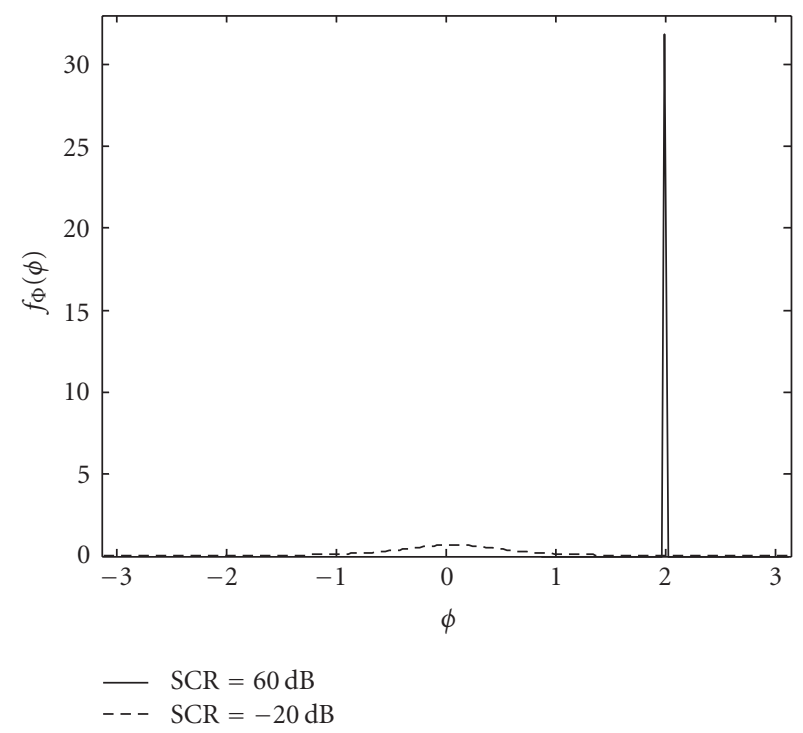

FIGURE 6: pdf of the interferometric phase in presence of a moving target such that $\phi_{v}=2.0 \mathrm{rad}, \mathrm{CNR}=10 \mathrm{~dB}, \gamma_{c}=0.95$, and for a high value of SCR $(60 \mathrm{~dB})$ and a low value of SCR $(-20 \mathrm{~dB})$.

to the same values of CNR and $\gamma_{c}$ in Figure 4 . For lower values of SCR, neither a shift equal to $\phi_{v}$ nor the preservation of pdf values is guaranteed, as shown in Figures 5 and 6, and this circumstance must be taken into proper account if one wants to correctly detect moving targets and retrieve their velocities in along-track InSAR applications.

\section{MULTICHANNEL ACROSS-TRACK SAR INTERFEROMETRY: HEIGHT RECONSTRUCTION}

Across-track SAR interferometric (InSAR) systems allow the reconstruction of the height profile of the earth surface [1]. It consists of the transformation of the modulo- $2 \pi$ (wrapped) interferometric phase signal into a (unwrapped) phase signal whose values are not limited to the interval $(-\pi, \pi]$. As this unwrapped phase signal is related to the height profile of the earth surface $[1,2]$, this operation allows recovering the terrain topographic map. It should be noted that the unwrapping problem is ill-posed, since it admits an infinite number of solutions $[12,13]$.

Recently, two methods for overcoming this limitation, restoring the solution uniqueness by exploiting different data sets acquired with frequency diversity, have been proposed $[12,13,14]$. The unknown terrain profile is reconstructed from the knowledge of multiple wrapped interferometric phase (statistically independent) signals obtained with different working frequencies of the SAR system. They are based on maximum likelihood (ML) estimation techniques, and the multifrequency interferograms are obtained by subband filtering interferometric images $[12,13]$ splitting the overall bandwidth as shown in Figure 7. The multiple interferogram needed to reconstruct the height profile can be obtained also starting from a multi-baseline system configuration [8] (see Figure 8). We will refer in the following to multifrequency and/or multi-baseline configuration as multichannel configuration.

The first technique $[12,13]$ is based on classical estimation techniques, in particular, on maximum likelihood (ML) estimation, and can be implemented in a strictly local way [12] or by exploiting deterministic contextual information consisting in the approximation of the height surface through local planes [13]. The extension of the strictly local technique [12] to take into account deterministic contextual information [13] allows better reconstructions also in the presence of nonnegligible noise and by using reducedbandwidth systems [13].

The second technique [14] is based on Bayesian estimation techniques, in particular, on maximum a posteriori (MAP) estimation. Its implementation allows to overcome some limitations of ML multichannel approaches, and allows to reduce the number of interferograms with respect to the above-mentioned ML algorithms to obtain reliable height estimation. In the following, we describe in more detail the Bayesian technique.

\subsection{Multichannel maximum a posteriori estimation of height profiles}

Consider a discrete (lexicographically ordered) twodimensional (2D) point lattice $L=\{k, k=1, \ldots, M \times P\}$ on the ground, and let $\mathbf{h}=\left[\begin{array}{lllll}h_{1} & h_{2} & \cdots & h_{M \times P}\end{array}\right]^{T}$ be the corresponding ground elevation values. Consider now an InSAR (interferometric synthetic aperture radar) system and let $\phi_{k n}$ be the wrapped phase value measured at the lattice point $k$ and at the working frequency $v_{n}=c / \lambda_{n}$, where $c$ is the speed of the light (we assume here that the ground scene is observed with $N$ different sensors working at $N$ different frequencies). The wrapped phase values $\phi_{k n}$ relative to the position $k$ and frequency $\nu_{n}$ can be structured and ordered in the following way: let $\boldsymbol{\Phi}_{k}=\left[\begin{array}{llll}\phi_{k 1} & \phi_{k 2} & \cdots & \phi_{k N}\end{array}\right]^{T}$ be the vector of the wrapped phases measured at $k$ at the $N$ different working frequencies, let $\boldsymbol{\Phi}=\left[\begin{array}{llll}\boldsymbol{\Phi}_{1}^{T} & \boldsymbol{\Phi}_{2}^{T} & \cdots & \boldsymbol{\Phi}_{M \times P}^{T}\end{array}\right]^{T}$ be the vector collecting all available wrapped phase values (multifrequency interferograms), and let $\mathbf{h}$ be the vector of the unknown height values. The multi-baseline case can be easily derived by the following development by varying $d$ in place of $\lambda$.

Considering the statistical description of the phase noise samples, the mapping between phase values and height values, and the hypothesis of statistical independence between the phase noise samples, the likelihood function of the height values, once all values of wrapped phase have been measured, turns out to be [14]

$$
F_{m f}(\boldsymbol{\Phi} \mid \mathbf{h})=\prod_{k=1}^{M \times P} \prod_{n=1}^{N} f_{\Phi}\left(\phi_{k n} \mid h_{k} ; \lambda_{n}, d, \gamma_{k n}\right),
$$

where $\gamma_{k n}$ is the coherence coefficient at position $k$ and frequency $v_{n}=c / \lambda_{n}$, and $f_{\Phi}(\cdot)$ is given by (6) considering $h_{k}$ as unknown once that $\phi_{k n}$ has been measured. We refer to (16) as the multichannel likelihood function and to (6) as the single-channel likelihood function. 

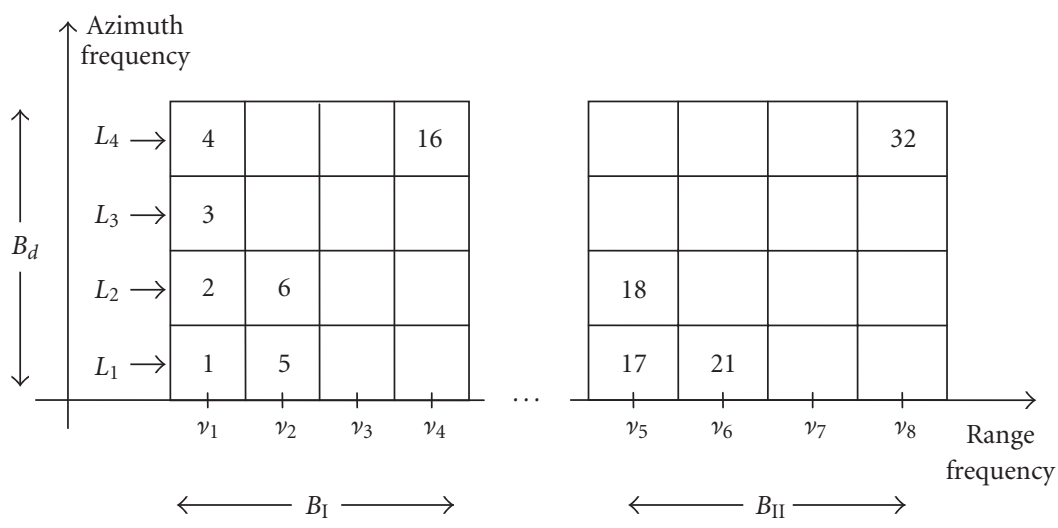

FIgURE 7: Partition of the dual band spectrum of a hypothetical SAR interferometric system. The two bands $B_{I}$ and $B_{I I}$ are subband filtered into $N_{r}=8$ range subbands (central frequencies $\left.\nu_{1}, v_{2}, \ldots, v_{8}\right)$, and the Doppler band $B_{d}$ into $N_{a}=4$ azimuth looks $\left(L_{1}, L_{2}, L_{3}, L_{4}\right)$. Each $\left(n=1, \ldots, N=N_{a} \times N_{r}=32\right)$ identifies a portion of the 2D frequency domain not overlapping with the others. Absence of overlapping guarantees the statistical independence of interferograms [13].



FIGURE 8: Multi-baseline (in particular, dual-baseline) across-track SAR interferometric system.

In terms of the MAP approach, the unknown image is a random vector $\mathbf{H}$ with an assigned a priori statistical distribution. In our case, being the unknown image representative of the elevation of a geographic area, a strong contextual pixel information is very likely to be. In particular, we assume that the unknown image can be modeled as a Markov random field (MRF) [15], a general image model able to represent contextual pixel information extending the 1D Markov property to the 2D case. In particular, we choose a Gaussian Markov random field (GMRF) [16] as model, whose expression, say it $g_{H}$, is

$$
\begin{aligned}
g_{H}(\mathbf{h} ; \boldsymbol{\sigma}) & =\frac{1}{Z(\boldsymbol{\sigma})} \exp \{-E(\mathbf{h} ; \boldsymbol{\sigma})\} \\
& =\frac{1}{Z(\boldsymbol{\sigma})} \exp \left\{-\sum_{k=1}^{M \times P} \sum_{j \in N_{k}}\left[\frac{\left(h_{k}-h_{j}\right)^{2}}{2 \sigma_{k j}^{2}}\right]\right\},
\end{aligned}
$$

where $Z(\boldsymbol{\sigma})=\int \exp \{-E(\mathbf{h} ; \boldsymbol{\sigma})\} d \mathbf{h}$ is the so-called partition function [16], $E(\cdot)$ is the energy function, $\sigma$ is the hyperparameter vector [16], $N_{k}$ is the neighbourhood system of $k$ th pixel [15] (usually, the 8 pixels around the $k$ th one), and $\sigma_{k j}$ are the single hyperparameters. The local GMRF model in (17) well adapts to describe the image local nature, by tuning the hyperparameters, leading to a powerful and general model, well suited to represent a wide class of height profiles.

These hyperparameter values have to be estimated from the same interferograms, through an ML estimation. This estimation problem is not trivial, and requires the adoption of approximations, as the use of the MPL (maximum pseudolikelihood) [19] to overcome the problems related to the use of the multivariate partition function $Z(\sigma)$ [15]. Finally, the expectation-maximization (EM) algorithm [16] allows to formulate the expression of the hyperparameter estimation update at $(i+1)$ th iteration:

$$
\begin{gathered}
\sigma_{k}^{2}(i+1)=E\left[\frac{\sum_{j \in N_{k}}\left(h_{k}-h_{j}\right)^{2}}{9} \mid \boldsymbol{\Phi}, \sigma_{k}(i)\right], \\
\sigma_{k}^{2}(i+1) \underset{i \text { large }}{\longrightarrow} \hat{\sigma}_{k}^{2},
\end{gathered}
$$

where the expected value is evaluated on random variable $H_{k}$ and the factor 9 in the denominator is resulting from the average over the chosen neighbourhood, formed by 9 pixels. For its evaluation, thanks to ergodicity of the a posteriori distribution [15], we can approximate this expected value by "time" averaging, sampling from the a posteriori distribution, which is itself an MRF, so that a Gibbs sampler [15] can be used for generating its samples. In particular, we use a Metropolis [19] version of this algorithm.

Once that the hyperparameter vector has been estimated, say it $\hat{\boldsymbol{\sigma}}$, the MAP estimation can be formulated as

$$
\begin{aligned}
\widehat{\mathbf{h}}_{\mathrm{MAP}} & =\underset{\mathbf{h}}{\operatorname{argmax}} f_{\text {Post }}(\mathbf{h} \mid \boldsymbol{\Phi}) \\
& =\underset{h}{\operatorname{argmax}} \ln F_{m f}(\boldsymbol{\Phi} \mid \mathbf{h}) g_{\mathbf{H}}(\mathbf{h} ; \hat{\boldsymbol{\sigma}}),
\end{aligned}
$$


where $f_{\text {Post }}(\mathbf{h} \mid \mathbf{\Phi})$ is the a posteriori joint pdf of the unknown image, and the "hat" denotes the estimated values. Then, the solution procedure essentially consists of two steps: (i) ML estimation $(\hat{\boldsymbol{\sigma}})$ of the hyperparameter vector $\boldsymbol{\sigma}$; (ii) MAP estimation $\left(\hat{\mathbf{h}}_{\mathrm{MAP}}\right)$ of the actual realization of height profile process $\mathbf{H}$.

For the MAP estimation, we use the simulated annealing (SA) [19] algorithm. In order to avoid excessive timeconsuming a semideterministic approach, which guarantees a reliable convergence, is adopted. Since the knowledge of the hyperparameter map is effective to avoid local minima in the image formation process, we follow this strategy: after hyperparameter estimation, we initialize the reconstruction algorithm with a high probability image sample, generated during the parameter estimation step, and starting from it we get the solution by ICM (iterated conditional modes) algorithm [19], which is deterministic, and then very fast.

\section{MULTICHANNEL ALONG-TRACK SAR INTERFEROMETRY RANGE-VELOCITY ESTIMATION}

Along-track synthetic aperture radar interferometry (ATInSAR) is a technique used to detect moving objects (ocean surface [3], ships, vehicles on ground [20]) by means of two (or more) SAR antennas mounted on the same platform flying along a linear trajectory at constant velocity $\mathbf{v}_{p}$, as shown in Figure 2, and to recover their range velocities. As already shown in by (4), the noise-free nominal interferometric phase is related to the range (orthogonal and coplanar to the platform motion direction) component of the object velocity.

The moving object detection capability and the accuracy of the velocity estimation depend essentially on the target range velocity and on three parameters: SCR, CNR, and clutter coherence $\gamma_{c}$. For detecting the moving targets in the SAR images, a possible strategy amounts to compare the interferometric phase with a threshold. Of course, at different choices of the threshold correspond different false-alarm probability $\left(P_{F A}\right)$ and detection probability $\left(P_{D}\right)$. For estimating the target range velocity, in AT-InSAR applications, only one or very few pixels can be exploited, differently from the acrosstrack case, where contextual relations between neighbouring pixels help to reconstruct the height profiles. In case of low SCR, a single value of the interferometric phase, which is noisy and characterized by a polarized pdf (see pdf in Figure 5), does not guarantee faithful detection and estimation.

In the following section, we show how the use of a multichannel, which also in this case can be multifrequency and/or multi-baseline, will help to find a reliable solution to this problem. As far as the multifrequency configuration is considered, the choice of the different frequencies is the same adopted in the across-track case (see Figure 7). As far as the multi-baseline configuration is concerned, the multiple antennas have to be positioned along the flight trajectory as shown in Figure 9.



FIGURE 9: Multi-baseline (in particular, dual-baseline) along-track SAR interferometric system.

The method adopted for the velocity estimation is based on an ML approach and exploit multichannel interferometric phase.

\subsection{AT-InSAR moving target detection capabilities}

Consider a point target moving with constant velocity $\mathbf{v}=$ $u_{x} v_{p} \mathbf{x}+u_{r} v_{p} \mathbf{r}$, where $u_{x}$ and $u_{r}$, with $\left|u_{x}\right|,\left|u_{r}\right| \ll 1$, are the dimensionless target velocity components along the $\mathbf{x}$ (azimuth) and $\mathbf{r}$ (slant range) coordinates, scaled to the platform velocity $v_{p}=\left|\mathbf{v}_{p}\right|$.

As shown in Section 2, the range velocity affects the interferometric phase which in the ideal noise-free case is given by the deterministic quantity $\phi_{v}=\left\langle(4 \pi b / \lambda) u_{r}\right\rangle_{2 \pi}$, while in the real case becomes a random variable $\Phi$ distributed according a pdf depending on several parameters:

$$
f_{\Phi}\left(\phi \mid u_{r} ; \lambda, b, \gamma_{c}, \text { SCR, CNR }\right) .
$$

Of course, in absence of a moving target $\left(u_{r}=0\right.$, and SCR $=$ $0)$ (20) reduces to (5) with $\phi_{0}=0$, and is available in analytical form. It has to be noted that both pdfs (20) and (5) are defined in the interval $(-\pi, \pi]$ highlighting the wrapped nature of the available actual interferometric phase.

A moving target can be detected by comparing the interferometric phase $\phi$ with a threshold $\phi_{T}$, and this threshold has to be fixed in the interval $(-\pi, \pi]$. Starting from the available pdfs relative to the hypotesis of presence and absence of moving target, we can evaluate the detection and false-alarm probability in the following way:

$$
\begin{aligned}
P_{D}= & \int_{-\pi}^{-\phi_{T}} f_{\Phi}\left(\phi \mid u_{r} ; \lambda, b, \gamma_{c}, \mathrm{SCR}, \mathrm{CNR}\right) d \phi \\
& +\int_{\phi_{T}}^{\pi} f_{\Phi}\left(\phi \mid u_{r} ; \lambda, b, \gamma_{c}, \mathrm{SCR}, \mathrm{CNR}\right) d \phi, \\
P_{F A}= & 2 \int_{\phi_{T}}^{\pi} f_{\Phi}\left(\phi \mid 0 ; \lambda, b, \gamma_{c}, 0, \mathrm{CNR}\right) d \phi .
\end{aligned}
$$


The plot of some pdfs relative to a given value of velocity such that $\phi_{v}=2(\mathrm{rad})$, to $\gamma_{c}=0.95$, to SCR $=0,10,20(\mathrm{~dB})$, and $\mathrm{CNR}=0,10,20(\mathrm{~dB})$, and the relative $P_{D}$ are shown in Figure 10.

The detection probability appears more sensitive to SCR than to CNR values. For values of $\mathrm{SCR}=10$ and $20 \mathrm{~dB}$, $P_{D} \approx 1$ for threshold values lower than 1 , for SCR $=0$ [dB] (the powers reflected from the moving target and the clutter are equal in the resolution cell), a threshold lower than 0.5 still guarantees $P_{D} \approx 1$.

The plot of the pdf relative to absence of moving target obtained for $\gamma_{c}=0.95$, and $\mathrm{CNR}=0,10,20(\mathrm{~dB})$, and the relative $P_{F A}$ are shown in Figure 11 .

If one wants to keep low values of $P_{F A}$, for instance, equal to $P_{F A}=0.1$, a threshold equal to $0.6,0.9$, and 2.2 (rad) must be used for the cases relative to $C N R=20,10$, and $0(\mathrm{~dB})$, respectively. In correspondence of these values, $P_{D} \approx 1$ for $\mathrm{SCR}=20(\mathrm{~dB}), P_{D} \geq 0.95$ for SCR $=10(\mathrm{~dB})$, and $P_{D}$ collapses toward values $0.1 / 0.2$ for SCR $=0(\mathrm{~dB})$.

The performance of the detection process is, as expected, better for high values of SCR, that is, when the moving targets power is significantly larger than the clutter power. For moving targets mingling with the background clutter, the detection capability worsens, so that if one wants low values of $P_{F A}$, the $P_{D}$ can decrease to very low values, not consistent with the applications.

It has to be considered that such performance is obtained starting from a single interferometric phase value. Exploiting multichannel information and a proper processing of the available data phase vector, such performance can be significantly improved.

\subsection{AT-InSAR moving target range-velocity estimation}

The next step after the detection of the moving target is the estimation of its normalized range velocity $u_{r}$. It has to be remembered that phase values outside interval $(-\pi, \pi)$ wrap $\bmod (2 \pi)$, so that such values are indistinguishable from the ones differing for $2 \pi$ multiples, and the same holds for the corresponding velocity values. It results that the maximum range-velocity value that can be unambiguously detected is given by

$$
\pm \pi=\frac{4 \pi b}{\lambda} u_{r, \max } \Longrightarrow\left|u_{r, \max }\right|=\frac{\lambda}{4 b}
$$

We propose a new method for recover the range velocity, whose values can be ambiguous, based on the use of a multichannel along-track SAR interferometer exploiting an ML approach.

Following the same approach outlined in $[12,13]$, the SAR complex images can be subband filtered at different central frequencies in order to generate samples of the interferometric phase at different wavelength $\lambda_{n}(n=1, \ldots, N)$, and/or a multi-baseline configuration can be adopted, obtaining different baseline $b_{m}(m=1, \ldots, M)$. Main effect of the use of multichannel interferograms is the velocity ambiguity suppression. In fact, at different wavelength and/or at different baseline, as it can be inferred by (22), the ambiguous velocity changes. Properly combining this "multichannel" phase information relative to different frequencies and/or different baselines, it can be highlighted [12] that the maximum likelihood (ML) estimation of the target velocity is not ambiguous.

This approach is based on the construction of the multichannel likelihood function that can be obtained by multiplying the likelihood functions corresponding to the central frequency of the subbands and to the different baselines:

$$
L\left(u_{r}\right)=\prod_{n, m} f_{\Phi}\left(\phi_{n, m} \mid u_{r} ; \lambda_{n}, b_{m}, \gamma_{c}, \mathrm{SCR}, \mathrm{CNR}\right),
$$

where $\phi_{n, m}$ represents the wrapped phase values relative to the frequency $c / \lambda_{n}$, and to the baseline $b_{m}$. The factorization (23) comes from the statistical independence of the multichannel interferograms.

The ML estimation of the range velocity from the multichannel data is finally given by

$$
\widehat{u}_{r}=\underset{u_{r}}{\operatorname{argmax}} L\left(u_{r}\right)
$$

\section{NUMERICAL RESULTS}

\subsection{Multichannel MAP-MRF height reconstructions}

We test the performance of the MRF-MAP method presented in Section 3.1 for a simulated height profile, considering the DEM of the Isolation Peak (Colorado), shown in Figure 12a. The used interferograms presented in Figures 12b and $12 \mathrm{c}$ are, respectively, simulated at $N_{r}=2$ different frequencies $\left(\nu_{1}=5 \mathrm{GHz}\right.$ and $\left.\nu_{2}=9 \mathrm{GHz}\right)$, and the coherence value is $\gamma=0.85$ for both frequencies.

For each working frequency, $N_{a}=4$ looks are generated by bandpass filtering of the azimuth spectrum, so allowing to generate $N=N_{r} \times N_{a}=8$ independent interferograms. The adopted system parameters, including the coherence value, are those of the SRTM mission [21].

The profile presents areas where the height discontinuities correspond to about $10.4 \pi$ at $5 \mathrm{GHz}$ and $18.8 \pi$ at $9 \mathrm{GHz}$; moreover, these jumps do not correspond to isolated points but they correspond to connected contours, so that it is impossible to unwrap such a profile using a (conventional) single-channel interferogram. The hyperparameter map estimated from the DEM is shown in Figure 12d, while the hyperparameter map estimated from the noisy interferograms is shown in Figure 12e. The hyperparameter map in Figure 12d which cannot be obtained in real applications because the DEM which is not known (it is indeed the unknown of the problem) can be used as a benchmark for the hyperparameter map reported in Figure 12e. The almost-perfect accordance between the two maps confirms the high reliability of the ML estimation of hyperparameters. The MAP height estimation is finally shown in Figure $12 \mathrm{e}$, and it highlights the quality of the height profile reconstruction by a comparison with the reference profile given in Figure 12a. 


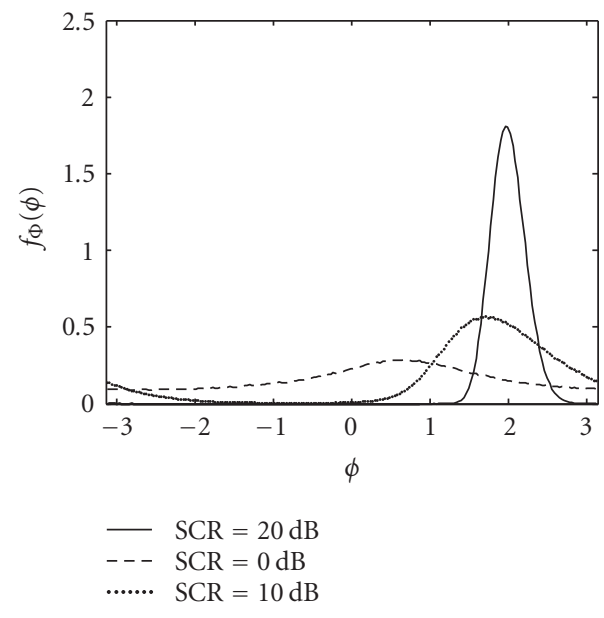

(a)

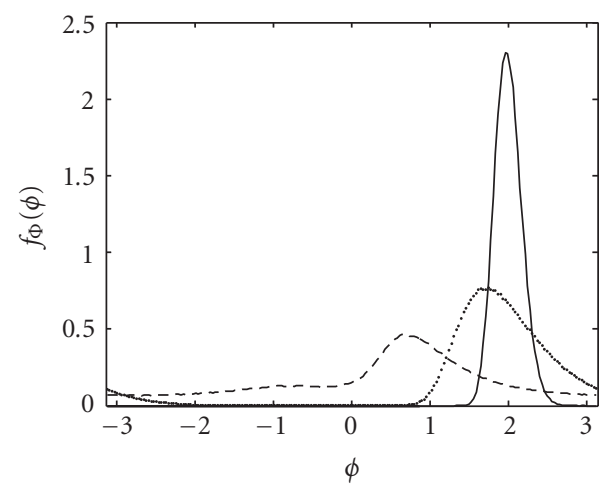

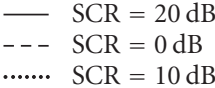

(c)

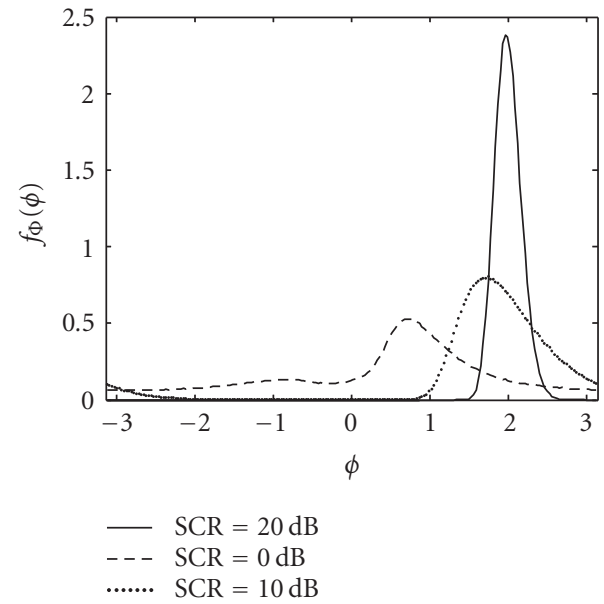

(e)

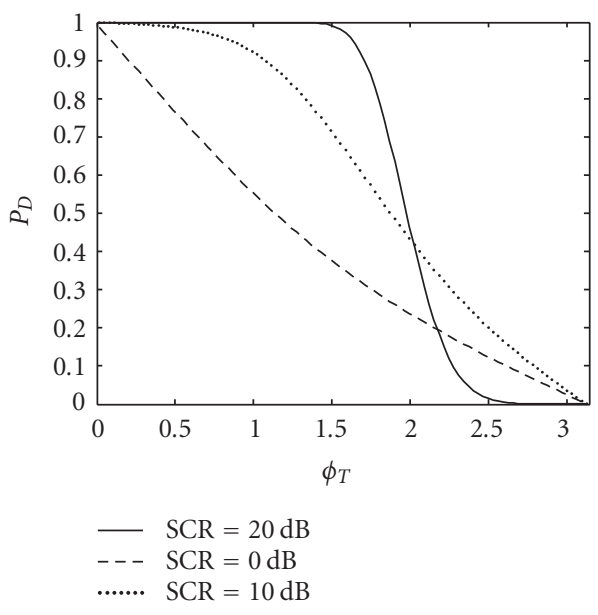

(b)



(d)

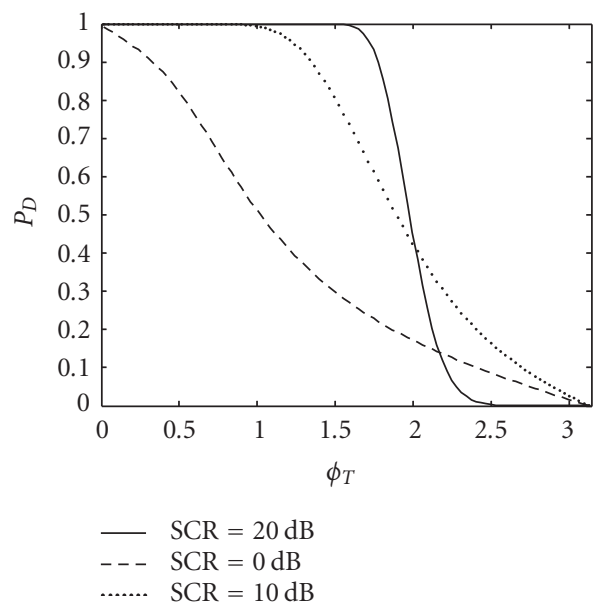

(f)

Figure 10: (a), (c), and (d) pdfs relative to a given value of velocity such that $\phi_{v}=2(\mathrm{rad})$, to $\gamma_{c}=0.95$, to $\mathrm{SCR}=0,10,20(\mathrm{~dB})$, and $\mathrm{CNR}=0,10,20(\mathrm{~dB})$, and $(\mathrm{b}),(\mathrm{d})$, and (f) the relative detection probabilities. 


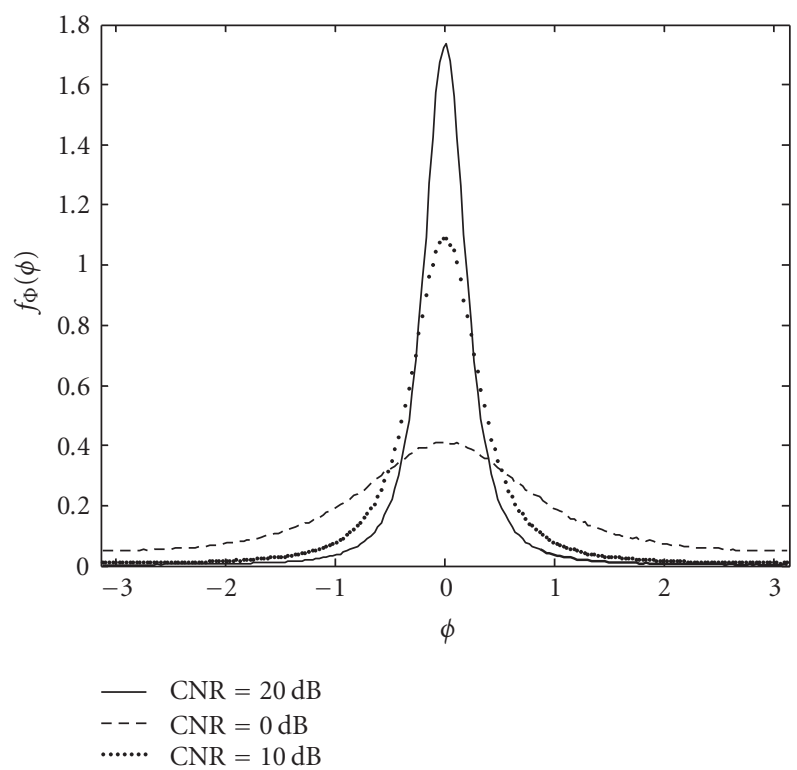

(a)



(b)

FIGURE 11: (a) pdfs relative to absence of moving target for $\gamma_{c}=0.95, \mathrm{CNR}=0,10,20(\mathrm{~dB})$, and (b) relative false-alarm probabilities.

\subsection{Multichannel ML range-velocity estimation}

We present some results relative to the application of the multichannel-based method to a hypothetical airborne system. The chosen central working frequency is $5.3(\mathrm{GHz})$ (the corresponding wavelength is $\left.\lambda_{c}=0.0566(\mathrm{~m})\right)$, and the velocity of the platform is fixed to $v_{p}=200(\mathrm{~m} / \mathrm{s})$. We consider and discuss three system configurations:

(a) total bandwidth $B=100(\mathrm{MHz})$, single baseline $b=$ $0.25(\mathrm{~m})$

(b) total bandwidth $B=400(\mathrm{MHz})$, single baseline $b=$ $0.25(\mathrm{~m})$

(c) total bandwidth $B=50(\mathrm{MHz})$, dual baseline $b_{1}=$ $0.25(\mathrm{~m}), b_{2}=0.42(\mathrm{~m})$.

In case (a), the maximum unambiguous range velocity is given by $\left|u_{r, \max }\right|=\lambda / 4 b=0.056$. In terms of absolute velocity, this value corresponds to $\left|v_{r, \max }\right|=\left|u_{r, \max }\right| \times v_{p}=$ $11.32(\mathrm{~m} / \mathrm{s})=40.75(\mathrm{~km} / \mathrm{h})$. Such result means that by using a single-channel along-track interferometer (one baseline, one frequency), it is not possible to infer the velocity of a target moving with a range velocity larger than $\left|u_{r \text {, max }}\right|$, even if the key parameters assume very favourable values (e.g., large values of SCR and $\gamma_{c}$, and low values of CNR). A target with a large radar cross-section as a car or a truck $\left(\sigma_{T} \geq 100\left(\mathrm{~m}^{2}\right)\right.$, then providing a large SCR $)$ moving at a range velocity about equal to $\pm 2\left|u_{r, \max }\right|= \pm 81.5(\mathrm{~km} / \mathrm{h})$ is seen as still clutter (such value of velocity wraps on a nominal interferometric phase equal to 0 ).

For this reason, we subband partition the available system bandwidth into $N_{a}=8$ looks and $N_{r}=4$ range subbands, so allowing to generate $N=N_{r} \times N_{a}=32$ independent interferograms. Such interferograms are not characterized by very different central frequencies so a strong capacity is not expected to avoid the velocity ambiguity [13]. To run the numerical experiments, we fix the following values: $\gamma_{c}=$ 0.95 (typical values for AT-InSAR application are larger than $0.95)$, SCR $=10(\mathrm{~dB})$ (usually for airborne applications SCR values are larger, but in this case, we considered the effect of multilooking), and $\mathrm{CNR}=20[\mathrm{~dB}]$ (such value can be much larger in airborne applications). The range velocity to be estimated has been chosen $u_{r}=0.08$, external to the unambiguous interval. Such case cannot be treated with conventional single-baseline AT-InSAR. The results of the estimation are summarized in the first row of Table 1, where in the left column is reported the percentage of faithful estimation (errors lower than 3\%), and in the right case the percentage of totally erroneous estimation. It has to be noted that the estimation method is potentially very accurate (the variance of the noise is not very high for such value of SCR, CNR, and $\gamma_{c}$ ), so that the erroneous results are due to the estimation of ambiguous values: when the method works well, estimate is very accurate and close to the right solution; when the method does not work, estimate is very accurate and close to an ambiguous value of velocity. For this reason, a column reporting medium quality estimation is absent in the table.

The range velocity is correctly estimated in about the $50 \%$ of the considered cases, denoting the usefulness of the introduction of multichannel information. The percentage of correct estimation would have been $0 \%$ in a single-channel configuration.

Consider now case (b), where a larger bandwidth is available for the subband partition. We filter out $N_{a}=8$ looks and $N_{r}=4$ range subbands, so allowing to generate $N=$ $N_{r} \times N_{a}=32$ independent interferograms. The chosen range velocity to be estimated is $u_{r}=0.08$, as in the previous case 


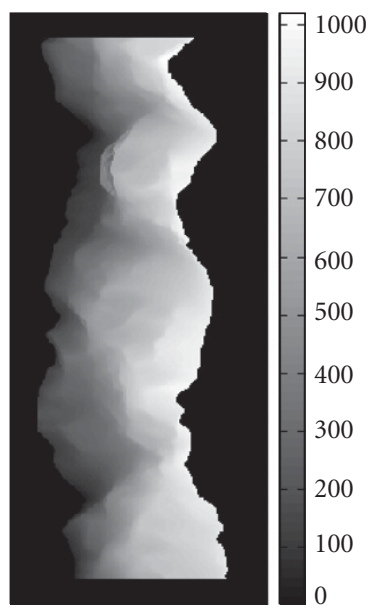

(a)

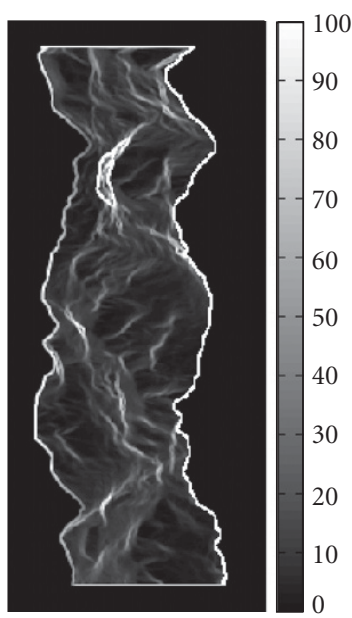

(d)

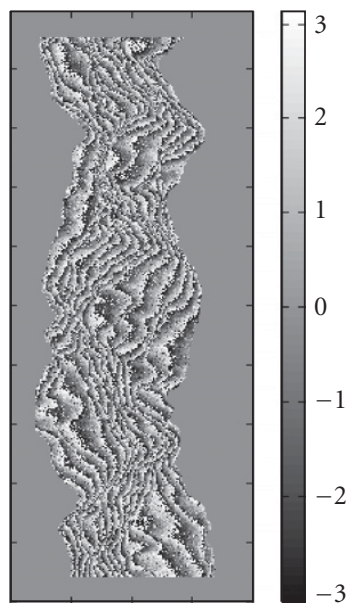

(b)

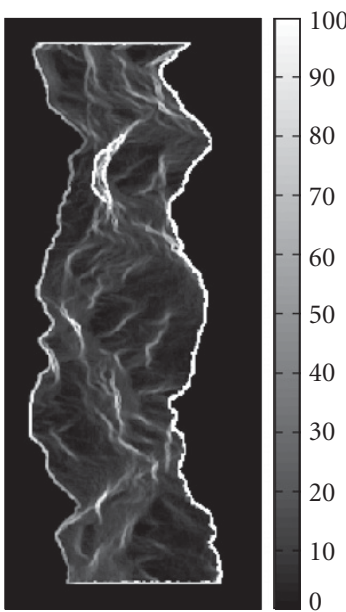

(e)

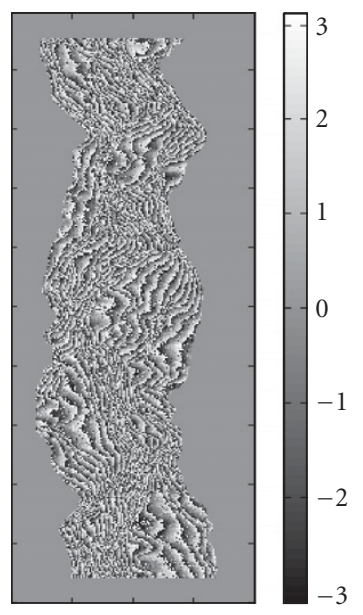

(c)

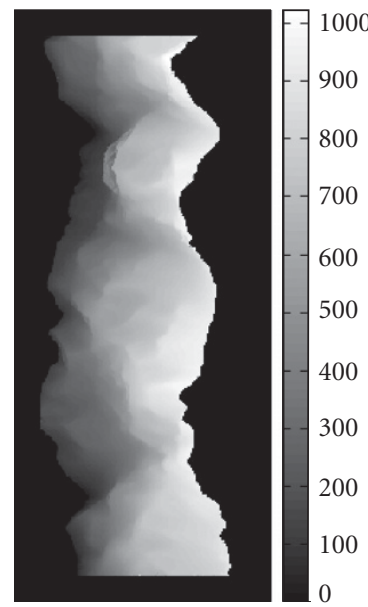

(f)

FIGURE 12: (a) Reference profile, (b) noisy ( $\gamma=0.85)$ interferogram at $5 \mathrm{Ghz}$ (C band), (c) noisy $(\gamma=0.85)$ interferogram at $9 \mathrm{Ghz}$ (X band), (d) reference hyperparameter map, (e) hyperparameter map estimation from noisy interferograms, and (f) MAP reconstruction.

TABLE 1: Range-velocity estimation performance. SCR $=10(\mathrm{~dB})$, $\mathrm{CNR}=20(\mathrm{~dB}), \gamma_{c}=0.95$. Velocity to be estimated $u_{r}=0.08$, out of the unambiguous interval $( \pm 0.056)$ of the InSAR system at $f_{c}=5.3(\mathrm{GHz})$, and $b=0.25(\mathrm{~m})$. Case (a) total bandwidth $B=$ $100(\mathrm{MHz})$, single baseline $b=0.25(\mathrm{~m})$. Case (b) total bandwidth $B=400(\mathrm{MHz})$, single baseline $b=0.25(\mathrm{~m})$. Case $(\mathrm{c})$ total bandwidth $B=50(\mathrm{MHz})$, dual baseline $b_{1}=0.25(\mathrm{~m}), b_{2}=0.42(\mathrm{~m})$.

\begin{tabular}{lcc}
\hline Case & Correct estimation (\%) & Wrong estimation (\%) \\
\hline Case (a) & $50 \%$ & $50 \%$ \\
Case (b) & $68 \%$ & $32 \%$ \\
Case (c) & $100 \%$ & $0 \%$ \\
\hline
\end{tabular}

(a). The estimation results are reported in the second row of Table 1 . The range velocity is correctly estimated in about $68 \%$ of the considered cases, denoting the improved capacity deals with a set of central frequencies more different from each other than in case (a).
Finally, case (c) relative to a dual baseline configuration is considered. In this case, the available bandwidth has been reduced to $50 \mathrm{MHz}$, and a third antenna has been added in order to consider a dual-baseline $(M=2)$ system. In order to generate the multichannel interferogram, for each baseline, we filter out $N_{a}=8$ looks and $N_{r}=2$ range subbands, so allowing to generate $N=N_{r} \times N_{a}=16$ multifrequency independent interferograms. $M \times N=32$ channels are available also in this case. The chosen range velocity to be estimated is $u_{r}=0.08$, as in the previous cases (a) and (b). In this case there are two values for the unambiguous range velocity, given by $\left|u_{r, \max }^{(1)}\right|=\lambda / 4 b_{1}=0.056$, and $\left|u_{r, \max }^{(2)}\right|=\lambda / 4 b_{2}=0.033$. It has to be noted that the new set of interferograms relative to the larger baseline introduces more critical measures from the point of view of the ambiguity solution. Nonetheless, the estimation results reported in the second row of Table 1 show that the range velocity is correctly estimated in $100 \%$ of the considered cases, denoting 
the impressive capacity of a dual baseline system to estimate range velocity without incurring in ambiguous values of velocity.

One of the main consequences of an incorrect rangevelocity estimation is a significant displacement of the moving target in the SAR image. The estimated target velocities then can be exploited to relocate the moving objects in the right positions of the final SAR images.

\section{CONCLUSIONS}

Some techniques based on statistical estimation dealing with multichannel InSAR applications have been analyzed in this paper. It has been shown that the joint use of multichannel configurations and statistical solution techniques allows to widen the classes of height profiles and moving target velocity that can be reliably reconstructed. In particular, as far as the across-track case and the height profiles reconstruction are concerned, the proposed MAP approach incorporating the a priori statistical information of the height profiles through an MRF model has evidenced that it is not necessary to have at one's disposal a large number of channels to get reliable solutions. The same comments hold for the along-track case and the range-velocity estimation. The number of channels necessary to reach good performances is sufficiently low, thus allowing the practical application of the method.

Multichannel data, which can be obtained by wideband systems and/or by more interferometric pairs of SAR images of the same ground area, are necessary to avoid solution ambiguities and to improve estimation accuracy. For this reason, the performance analysis results of multichannel configurations are very important for future interferometric missions planning.

\section{REFERENCES}

[1] S. N. Madsen, H. A. Zebker, and J. Martin, "Topographic mapping using radar interferometry: processing techniques," IEEE Trans. Geosci. Remote Sensing, vol. 31, no. 1, pp. 246-256, 1993.

[2] R. Bamler and P. Hartl, "Synthetic aperture radar interferometry," Inverse Problems, vol. 14, no. 4, pp. R1-R54, 1998.

[3] R. M. Goldstein and H. A. Zebker, "Interferometric radar measurements of ocean surface currents," Nature, vol. 328, no. 6132 , pp. 707-709, 1987.

[4] R. E. Carande, "Dual baseline and frequency along-track interferometry," in Proc. IEEE International Geoscience and Remote Sensing Symposium (IGARSS '92), vol. 2, pp. 1585-1588, Houston, Tex, USA, May 1992.

[5] D. C. Ghiglia and M. D. Pritt, Two-Dimensional Phase Unwrapping: Theory, Algorithms, and Software, John Wiley \& Sons, New York, NY, USA, 1998.

[6] D. C. Ghiglia and L. A. Romero, "Robust two-dimensional weighted and unweighted phase unwrapping that uses fast transforms and iterative methods," Journal of the Optical Society of America A, vol. 11, no. 1, pp. 107-117, 1994.

[7] M. Costantini, "A novel phase unwrapping method based on network programming," IEEE Trans. Geosci. Remote Sensing, vol. 36, no. 3, pp. 813-821, 1998.
[8] A. Ferretti, C. Prati, and F. Rocca, "Multibaseline phase unwrapping for InSAR topography estimation," Il Nuovo Cimento, vol. 24, no. 1, pp. 159-176, 2001.

[9] J. Homer, I. D. Longstaff, Z. She, and D. Gray, "High resolution 3-D imaging via multi-pass SAR," IEE Proceedings Radar, Sonar and Navigation, vol. 149, no. 1, pp. 45-50, 2002.

[10] D. Massonnet, H. Vadon, and M. Rossi, "Reduction of the need for phase unwrapping in radar interferometry," IEEE Trans. Geosci. Remote Sensing, vol. 34, no. 2, pp. 489-497, 1996.

[11] A. Budillon, V. Pascazio, and G. Schirinzi, "Multi-channel along track interferometry," in Proc. IEEE International Geoscience and Remote Sensing Symposium (IGARSS '04), vol. 4, pp. 2601-2603, Anchorage, Alaska, USA, September 2004.

[12] V. Pascazio and G. Schirinzi, "Estimation of terrain elevation by multifrequency interferometric wide band SAR data," IEEE Signal Processing Lett., vol. 8, no. 1, pp. 7-9, 2001.

[13] V. Pascazio and G. Schirinzi, "Multifrequency InSAR height reconstruction through maximum likelihood estimation of local plane parameters," IEEE Trans. Image Processing, vol. 11, no. 12, pp. 1478-1489, 2002.

[14] G. Ferraiuolo, V. Pascazio, and G. Schirinzi, "Maximum a posteriori estimation of height profiles in InSAR imaging," IEEE Geoscience and Remote Sensing Letters, vol. 1, no. 2, pp. 66-70, 2004.

[15] S. Geman and D. Geman, "Stochastic relaxation, Gibbs' distributions, and Bayesian restoration of images," IEEE Trans. Pattern Anal. Machine Intell., vol. 6, no. 6, pp. 721-741, 1984.

[16] S. S. Saquib, C. A. Bouman, and K. Sauer, "ML parameter estimation for Markov random fields with applications to Bayesian tomography," IEEE Trans. Image Processing, vol. 7, no. 7, pp. 1029-1044, 1998.

[17] D. Just and R. Bamler, "Phase statistics of interferograms with applications to synthetic aperature radar," Applied Optics, vol. 33, no. 20, pp. 4361-4370, 1994.

[18] J. C. Curlander and R. N. McDonough, Synthetic Aperture Radar, Wiley Interscience, New York, NY, USA, 1991.

[19] S. Z. Li, Markov Random Field Modeling in Image Analysis, Springer, Berlin, 2001.

[20] H. Breit, M. Eineder, J. Holzner, H. Runge, and R. Bamler, "Traffic monitoring using SRTM along-track interferometry," in Proc. IEEE International Geoscience and Remote Sensing Symposium (IGARSS '03), vol. 2, pp. 1187-1189, Toulouse, France, July 2003.

[21] J. E. Hilland, F. V. Stuhr, A. Freeman, et al., "Future NASA spaceborne SAR missions," IEEE Aerosp. Electron. Syst. Mag., vol. 13, no. 11, pp. 9-16, 1998.

Alessandra Budillon received the Laurea degree (summa cum laude) in 1996, and the Ph.D. degree in 1999, in electronic engineering from the University of Napoli "Federico II," Napoli, Italy. From 2001 to 2004, she was an Assistant Professor at the Department of Information Engineering, the Second University of Nap, Aversa, Italy, and in November 2004, she joined the Department for Technologies, the University of

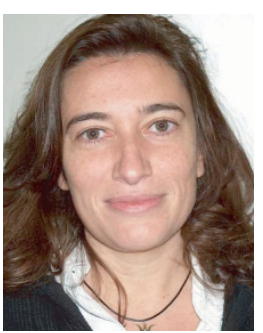

Napoli "Parthenope," Napoli, Italy, where she currently is an Assistant Professor. In 1998, she was a Visiting Student at the Department of Cognitive Science, MIT, Boston, USA. Her main research interests are in neural networks, statistical signal processing, and image processing, remote sensing. 
Giancarlo Ferraiuolo received the Laurea degree (summa cum laude) in electronic engineering from the Second University of Naples, Italy, in 2000, and the P.h.D. degree from the University of Napoli "Federico II," in 2004. In 2003, he spent a period as a Visiting Researcher in the Department of Electrical Engineering, Stanford University, Stanford, Calif. Since December 2004, he has been a researcher at the Department


of Electronic and Telecommunication Engineering, the University of Napoli "Federico II." His main scientific interests are in the field of statistical image formation for computed tomography and SAR applications.

Vito Pascazio graduated (summa cum laude) in 1986 from the University of Bari, Italy, in electronic engineering, and in 1990, he received the Ph.D. degree in electronic engineering and computer science from the Università di Napoli "Federico II," Italy. In 1990 he was first at IRECE-CNR, Napoli, Italy, and then, he joined the Università di Napoli "Parthenope," Italy, where he is presently a Full Professor of telecommuni-

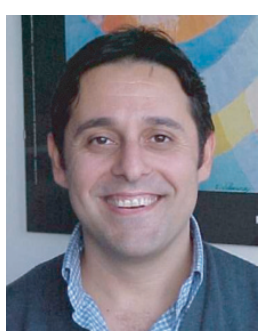
cations. In 1994-1995, he was a Visiting Scientist at the Laboratoire des Signaux et Systemes, Ecole Superieure d'Electricite, Gif sur Yvette, France, and in 1998-1999 at Université de Nice SophiaAntipolis, France. His main scientific interests include statistical signal processing, remote sensing, and image computing and processing, and he is the author of more than 90 technical papers on international journals and conference proceedings. Dr. Pascazio is a Member of IEEE.

Gilda Schirinzi graduated in electronic engineering in 1983 from the University of Napoli "Federico II." In the same year, she joined the Electronic Engineering Department as a Research Fellow. From 1985 to 1986, she was at European Space Agency, ESTEC, the Netherlands. In 1988, she joined Istituto di Ricerca per l'Elettromagnetismo e i Componenti Elettronici (IRECE), CNR, in Naples. In 1992,

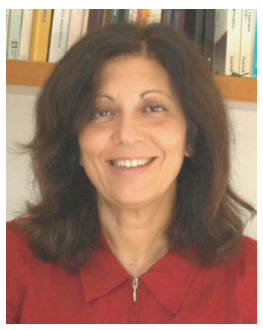
she was appointed Head of Electromagnetics Division, IRECE, and in 1997 she became a First Researcher at the same Institute. She is presently a Full Professor of telecommunications at the University of Cassino. Her main scientific interests are in the field of synthetic aperture radar (SAR) signal processing and coding. 